\title{
Correlation of Global Quantities at Material Characterization of Pressure-Sensitive Materials Using Sharp Indentation Testing
}

\author{
Carl F. O. Dahlberg, Jonas Faleskog and Per-Lennart Larsson *D \\ Unit of Solid Mechanics, Department of Engineering Mechanics, Royal Institute of Technology (KTH), \\ SE-10044 Stockholm, Sweden; carldahl@kth.se (C.F.O.D.); faleskog@kth.se (J.F.) \\ * Correspondence: plla@kth.se
}

check for

updates

Citation: Dahlberg, C.F.O.; Faleskog, J.; Larsson, P.-L. Correlation of Global Quantities at Material Characterization of Pressure-Sensitive Materials Using Sharp Indentation Testing. Lubricants 2021, 9, 29. https://doi.org/10.3390/ lubricants 9030029

Received: 18 January 2021

Accepted: 26 February 2021

Published: 3 March 2021

Publisher's Note: MDPI stays neutral with regard to jurisdictional claims in published maps and institutional affiliations.

Copyright: (c) 2021 by the authors. Licensee MDPI, Basel, Switzerland. This article is an open access article distributed under the terms and conditions of the Creative Commons Attribution (CC BY) license (https:// creativecommons.org/licenses/by/ $4.0 /)$.

\begin{abstract}
Correlation of sharp indentation problems is examined theoretically and numerically. The analysis focuses on elastic-plastic pressure-sensitive materials and especially the case when the local plastic zone is so large that elastic effects on the mean contact pressure will be small or negligible as is the case for engineering metals and alloys. The results from the theoretical analysis indicate that the effect from pressure-sensitivity and plastic strain-hardening are separable at correlation of hardness values. This is confirmed using finite element methods and closed-form formulas are presented representing a pressure-sensitive counterpart to the Tabor formula at von Mises plasticity. The situation for the relative contact area is more complicated as also discussed.
\end{abstract}

Keywords: indentation hardness; plastic contact; contact area; contact mechanics; finite-element method

\section{Introduction}

During a contact-related experiment, most often normal indentation or scratch testing, the obtained quantities can be used in order to determine the constitutive properties of the tested materials. It is also possible to determine field variables such as for example residual stresses. The most important properties determined during a normal indentation test are hardness, $H$, and the indentation load, $P$, versus indentation depth curve, $h$. The indentation hardness $H$ represents a measure of the mean contact pressure under the indenter and is calculated from the force necessary to push a rigid indenter into the material. An additional important parameter involved in the hardness calculation is the actual area of contact between the indenter and the material.

The resulting boundary value at normal indentation is very complicated and in order to arrive at a relation between the above mentioned indentation properties and material characteristics, advanced contact mechanics analyses are needed. When it comes to sharp indentation of elastic or standard (von Mises) elastoplastic materials such relations have been successfully derived and classical results by Sneddon [1], Tabor [2] and Johnson [3,4] have been verified and also further improved using modern computers and powerful numerical methods. The state-of-the-art as regards correlation of sharp indentation of standard elastoplastic materials will be outlined in some detail below.

First though, it should be mentioned that progress for other types of constitutive (material) behavior have been scarce or practically non-existent. This is especially so when it comes to general correlation aiming at theoretically based relations intended for material characterization. Indeed, excluding classical elastoplasticity such relations only exist for singular cases. It is the intention of the present investigation to remedy this shortcoming by performing a detailed analysis of sharp indentation of Drucker-Prager [5] elastoplastic materials developed for accounting for pressure-sensitivity at deformation.

Correlation of sharp indentation of classical elastoplastic materials is most often based on the results by Johnson [3,4] suggesting that the Johnson parameter

$$
\Lambda=E(\tan \beta) /\left(\left(1-v 2\left(\sigma_{\text {rep }}\right)\right.\right.
$$


governs the contact behavior. In Equation (1), $E$ is Young's modulus and $v$ is Poisson's ratio, $\beta$ is the angle between the (sharp) indenter and the undeformed surface, and orep is the material flow stress at a representative value of the effective (accumulated) plastic strain $\varepsilon p$. Three indentation levels are noticeable as depicted in Figure 1. Specifically, in Level I, $\Lambda<3$, all global properties can be derived from an elastic analysis. In level II, $3<\Lambda<30$, plastic deformation increases and both elastic and plastic properties are of importance for the outcome of the test. In this region $H \sim \ln \Lambda, \mathrm{cf}[3,4]$. In level III, $\Lambda>30$, plastic deformation dominates the contact situation which is a situation applicable to most engineering metals. Based on extensive experimental results, Tabor [2] proposed the relation

$$
H=C \sigma_{\text {rep }}
$$

where $C$ is a constant depending on the (sharp) indenter geometry. For a Vickers indenter, the values $C \approx 3$ and $\varepsilon p \approx 0.08$ was suggested in [2] while $C \approx 2.55$ and $\varepsilon p \approx 0.11$ was suggested by Atkins and Tabor [6] for a cone indenter with an angle of $22^{\circ}$ between the indenter and the undeformed surface.

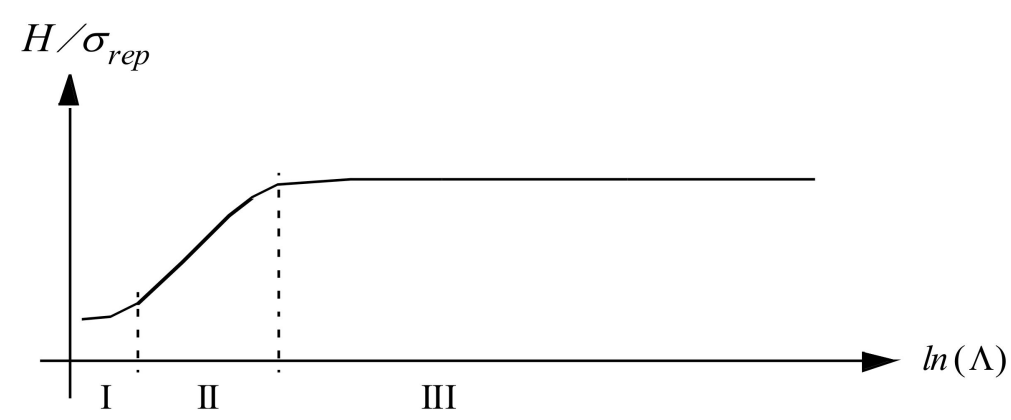

Figure 1. Sketch of the characteristic behaviour at indentation. The indentation hardness $H$ divided by the representative stress $\sigma r e p$ is plotted against the non-dimensional strain parameter $\Lambda$ suggested by Johnson $[3,4]$. The different levels at indentation are indicated.

Sharp indentation has during the last 30 years or so also been extensively investigated using numerical methods and in particular the finite element method (FEM). Important contributions in this context include Bhattachharya and Nix [7,8], Laursen and Simo [9], Giannakopoulos and co-workers [10,11], Mesarovic and Fleck [12,13], and Larsson [14]. For one thing, improvements of Equation (2) have been suggested especially for materials with irregular strain-hardening. However, at power law hardening plasticity, also the numerical studies above indicate that Equation (2) is sufficient for hardness predictions. For details, it was shown by Larsson [14] that at power law hardening

$$
\sigma_{\text {rep }}=\sigma\left(\varepsilon_{r}=0.18\right)
$$

for a cone indenter (with an angle of $22^{\circ}$ between the indenter and the undeformed surface), and using $C \approx 2.55$ in Equation (2), yielded very accurate results. Correspondingly, for Vickers and Berkovich indenters

$$
\sigma_{\text {rep }}=\sigma\left(\varepsilon_{r}=0.15\right)
$$

using $C \approx 2.8$ in Equation (2). It should be emphasized that the representative stresses in Equations (3) and (4) are relied upon throughout the present investigation.

The relative contact area $c^{2}$ is also a global indentation quantity of interest when correlation is at issue. This parameter is defined as

$$
c^{2}=A / A_{\text {nom }}
$$

where $A$ is the actual projected contact area at indentation and $A_{\text {nom }}$ is the nominal projected contact area resulting in neither sinking-in nor piling-up at the contact boundary. The 
parameter $c^{2}$ is also a function of material properties alone at fixed indenter geometry. Material characterization based on $c^{2}$ is, however, cumbersome at best as it is very much influenced by elastic effects, cf. e.g., [12-14], also at high values on the $\Lambda$-parameter in Equation (1), and a simple relation as for the hardness in Equation (2) is not relevant in a practical situation.

Accordingly, sharp indentation of classical elastoplastic materials is nowadays quite well understood, also in a quantitative manner, and for this reason indentation is a suitable tool for characterization of such materials. As indicated above, for other types of constitutive behavior progress is limited and indentation then becomes a comparative experimental method but details of the material characteristics, for example explicit values on material properties, cannot be determined in a reliable manner.

Drawing upon the theory outlined, an obvious candidate for extending the usefulness of sharp indentation for material characterization is pressure-sensitive elastoplastic materials. This is especially so in the case when pressure-sensitivity can be modeled in a fairly simple manner introducing a limited additional number of constitutive properties. In particular this concerns classical results for Drucker-Prager elastoplasticity [5] where in case of associative plastic flow only one additional material parameter is introduced; namely the pressure sensitivity factor $\alpha$.

Therefore, the present intention is to perform an analysis, as the one above for classical von Mises plasticity, but also accounting for pressure-sensitivity of the material. This feature has been investigated previously, cf. [15-19], and many aspects regarding level II indentation have been clarified. Furthermore, materials with a soft elastic response with or without plastic volume change have been investigated by Needleman et al. [20]. However, a detailed approach in the spirit of Tabor [2], devoted toward correlation in the rigid-plastic deformation stage, has to the author's knowledge not yet been achieved or even attempted. Accordingly, this is the aim of the present study. First of all then, analytical considerations provide the basic foundations for the structure of the equations in the same spirit as the corresponding ones for classical von Mises plasticity (Equations (1)-(4)). Second, the analytical achievements are verified and generalized by numerical (finite element) results pertinent to a wide range of material characteristics.

Concerning the constitutive specification of the pressure-sensitive material classical Drucker-Prager plasticity [5] is adhered to. Associativity of the flow rule is relied upon.

\section{Problem Formulation}

The problem at issue concerns conical and for discussion also pyramid indentation, see Figure 2, of pressure-sensitive elastoplastic materials. At conical indentation, a sharp rigid conical stylus with an angle of $22^{\circ}$, between the indenter and the undeformed surface, see Figure 2, is used. The angle $22^{\circ}$ is chosen as being the same as the corresponding angle at Vickers (sharp pyramid indenter with quadratic base) indentation. In the presentation below $h$ is the normal indentation depth in the $X_{2}$-direction (Cartesian coordinates are introduced in Figure 2) and $A$ is the normally projected contact area between indenter and material. Quasi-static conditions are assumed and the dimensions of the contact region are very small compared to the size of the indented material specimen. Based on these restrictions, the problem is self-similar with no characteristic length present and this ensures that the (normal) hardness as well as the ratio $h / \sqrt{ } A$ (where $\sqrt{ } A$ should be interpreted as a characteristic contact length) is constant during the loading sequence of an indentation test on homogeneous materials. Here and in the sequel the normal hardness is defined as

$$
H=P / A
$$

where $P$ is the normal contact load, see Figure 2. 

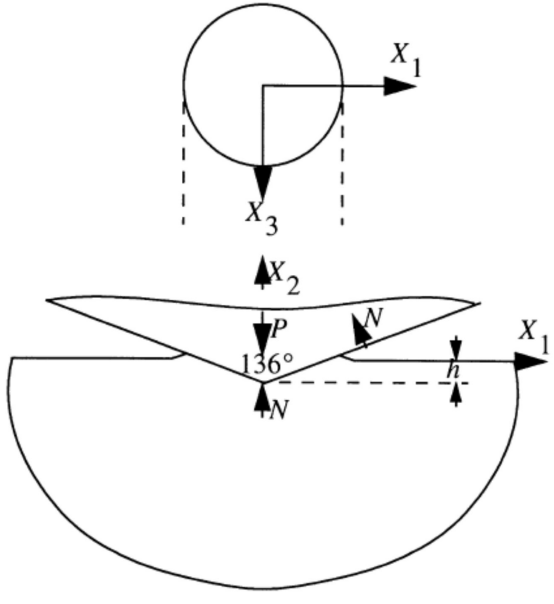

(a)
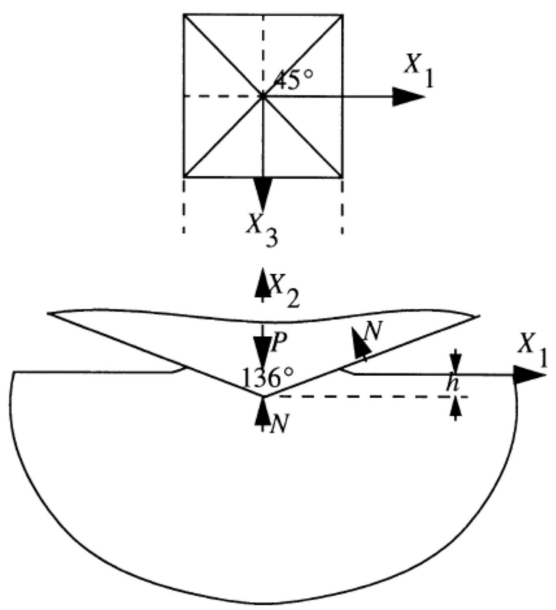

(b)

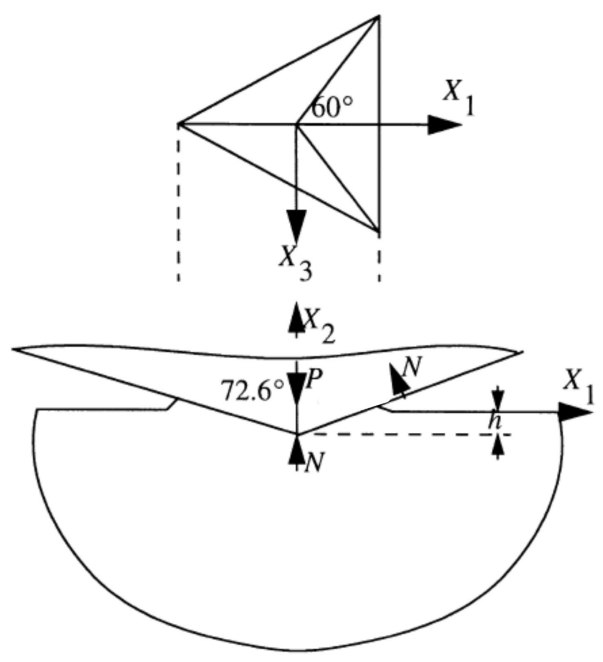

(c)

Figure 2. Schematics of the geometry of sharp indentation tests. Both top and side views are shown. Cartesian coordinates xi are introduced. (a) Cone indentation. (b) Vickers indentation. (c) Berkovich indentation.

Regarding the constitutive behavior, classical Drucker-Prager elastoplasticty is assumed to prevail with a yield condition

$$
\sigma_{e}+3 \alpha \sigma_{h}-\sigma\left(\varepsilon_{p}\right)=0
$$

where $\sigma_{e}$ is the von Mises effective stress, $\sigma_{h}$ is the hydrostatic stress, $\alpha$ is the pressure sensitivity factor, and $\sigma(\varepsilon p)$ is the flow stress in pure shear. Associativity of the flow rule is assumed in most situations but, to be detailed below, also incompressible plastic flow is analyzed and relevant results from the two cases are compared and discussed. Large deformation theory was relied upon due to the fact that such effects are significant at many contact problems, cf. e.g., [11,21]. At elastic loading, or unloading, a hypoelastic formulation of Hooke's law was relied upon. Kinematic hardening effects were not included in the analysis as only the loading part of the indentation test was of primary interest. The strain hardening behavior are based on a power law assumption according to

$$
\sigma(\varepsilon p)=\sigma_{0}(1+(\varepsilon p / \varepsilon 0))^{n}
$$


where $\sigma_{0}$ is the initial yield stress in pure shear, $\varepsilon p$ is as previously stated the effective plastic strain, and $\varepsilon 0$ and $n$ are material constants describing the plastic hardening.

In summary, the present analysis is restricted to quasi-static conditions and classical Drucker-Prager elastoplasticity and, as a result, strain rate effects are not included. Frictionless contact is assumed as a first approximation. The investigation is focused on level III contact conditions where the influence from elasticity on the hardness behavior is negligible.

\section{Theoretical Analysis}

In the case of classical von Mises plasticity it was shown by Johnson $[3,4]$ that at elasticplastic (level II) sharp indentation the material hardness is well described by the relation.

$$
\hat{H}=C_{1}+C_{2} \ln \Lambda
$$

where

$$
\hat{H}=H / \sigma_{\text {rep }}
$$

The derivation leading to Equation (9) rests on the similarity of the stress fields at sharp indentation and taking advantage of the analysis of expansion of a pressurized spherical cavity in an elastic-ideally plastic material. The solution to the latter problem (in the context of indentation testing) was originally proposed by Bishop et al. [22] and the effect from plastic strain-hardening is accounted for by the representative stress $\sigma_{\text {rep }}$. It has been shown with the aid of finite element calculations, cf. e.g., Larsson [23], that the constants $C_{1}$ and $C_{2}$ in Equation (9) are not strictly independent of the strain-hardening characteristics. However, as the present investigation concerns rigid plastic (level III) indentation this issue will not be dwelled upon further. It should be emphasized that at $\Lambda$-values pertinent to the transition between level II and level III indentation Equation (2) (nondimensionalized according to (10)) and Equation (9) obviously yield similar results.

The analysis by Johnson [3,4] was extended to pressure-sensitive materials, described by classical Drucker-Prager elastoplasticity, by Narasimhan [16] and further discussed by Zhang et al. [17] and Fornell et al. [18]. The resulting expression for the hardness (mean contact pressure) in the case of cone indentation yields

$$
H=H / \sigma_{y}=(1 / 3 \alpha)\left((1+2 \alpha)^{2} \bar{r}_{p y^{2 q}}-1\right)
$$

at ideally plastic material behavior where $q=3 \alpha /(1+2 \alpha)$. In (11),

$$
\bar{r}_{p}=r_{p} / a
$$

is the radius of the plastic zone, $r_{p}$, nondimensionalized by the radius of contact $a$. It should be noted that elastic effects are included in (11) only through $\bar{r}_{p}$.

As it stands, Equation (11) describes the hardness at level II cone indentation accounting for pressure-sensitivity through $\alpha$ but not for strain-hardening. In order to include the latter feature in the analysis and also determine the contact behavior at rigid plastic (level III) conditions, the following assumptions are made:

Level III conditions can be enforced by determining the value on the nondimensionalized plastic zone radius at rigid plastic contact, denoted as $\bar{r}_{p y}$. It should be noted that when (rigid) ideally plastic behavior is considered $\bar{r}_{p y}=\bar{r}_{p y}(\alpha)$.

Strain-hardening can be introduced as in case of von Mises plasticity in a standard manner solely by replacing $\sigma_{y}$ with the representative stress in Equation (3).

With (1) and (2) introduced into Equation (11) the relation

$$
H_{c}=H_{c}(\alpha)=H_{c} / \sigma\left(\varepsilon_{p}=0.18\right)=(1 / 3 \alpha)\left((1+2 \alpha)^{2} \bar{r}_{p y^{2 q}}-1\right)
$$


can be suggested describing the behavior of the hardness at level III cone indentation of Drucker-Prager elastoplastic materials accounting for strain-hardening. It should be emphasized that as a direct consequence of the structure of Equation (13) the influence on the material hardness from plastic strain-hardening and pressure-sensitivity is separable. This feature is of substantial importance for the practical usefulness of the present approach and will be carefully evaluated and, indeed, verified by FEM analyses to be described in detail below. In addition, explicit correlation formulae will also be determined based on the FEM results in order to verify and complement the theoretical results.

From Equation (13) it is obvious that an accurate determination of $\bar{r}_{p y}$ is fundamental to ensure a high predictive capability of this formula. Previously in [16-18], where the analysis is devoted to level II indentation, the corresponding results for classical elastoplasticity were used to determine the size of the plastic zone. This is however a very crude approximation and presently, finite element simulations were used to determine $\bar{r}_{p y}(\alpha)$ as described in more detail below.

It is of course of fundamental importance that the validity of the present analysis can be extended also to three-dimensional indentation problems, not the least so when it comes to the practical usefulness of the results. The most commonly known threedimensional indenters are the Vickers indenter (sharp indenter with a square base) and the Berkovich indenter (sharp indenter with a triangular base). It is known from previously, cf. e.g., $[10,11]$, that these two indenters behave very similar from a mechanical point of view. Indeed, the Berkovich indenter was developed for nanoindentation experiments but the geometry was chosen in order to replicate the hardness behavior of the Vickers indenter, [24]. For this reason, Berkovich and Vickers indentation is considered presently to yield the same result. In doing so, a third assumption can be made:

The ratio between cone and Berkovich (and Vickers) hardness value is independent of $\alpha$ and depends only on the choice of representative stress, pertinent to the different indenter geometries, as specified in Equations (3) and (4).

Based on this assumption, and the discussion in connection with Equations (3) and (4), the relation between the two hardness values yields

$$
H_{B} / H_{c}=1.10 \sigma\left(\varepsilon_{p}=0.15\right) / \sigma\left(\varepsilon_{p}=0.18\right)
$$

which obviously is the case also at classical von Mises plasticity. Accordingly, from Equations (13) and (14)

$$
H_{B}=H_{B}(\alpha)=H_{B} / \sigma\left(\varepsilon_{p}=0.15\right)=(1 / 3 \alpha)\left((1+2 \alpha)^{2} \bar{r}_{p y^{2 q}}-1\right)
$$

indicating a separable solution also for the (nondimensionalized) hardness value at Berko vich, and Vickers, indentation of Drucker-Prager pressure-sensitive materials. In (15), $\bar{r}_{p y}(\alpha)$ is taken to be the same as at cone indentation based on, for example, the results by Johnson [4] clearly showing that the size of the plastic zone is almost independent of the indenter geometry. The validity of Equation (15) will be investigated in more detail in a parallel study [25] and here only cone indentation is analyzed in detail.

Concerning the parameter $c^{2}$ defined in Equation (5), it is stated in the previous discussion that also this parameter can in particular situations be determined in closed form as a function of material parameters. Accordingly, in such situations it would be suitable for material characterization. However, as also mentioned above, elastic effects will severely influence this parameter and this feature makes $c^{2}$ much less useful when it comes to the determination of material properties. For completeness and as a basis for possible future analyses, explicit FEM results, pertinent to $c^{2}$, will also be presented below.

\section{Numerical Analysis}

The finite element analysis was performed using the commercial finite element package ABAQUS [26]. The finite element mesh is depicted in Figure 3. Here, only details close to the region of contact is shown but it should be emphasized that it was properly 
ensured that the entire meshed region was sufficiently large to avoid interference from the outer boundaries. The finite element mesh consisted of four-noded, axisymmetric elements being of hybrid type, i.e., the displacements were approximated with bilinear shape functions while the hydrostatic pressure attained a constant value in each element, in order to improve the convergence at close to incompressible deformation. The simulations were performed by controlling the displacement of the rigid conical indenter while keeping the outer boundary of the meshed area in a fixed position. Large deformation theory was used throughout the simulations with a single exception discussed below.

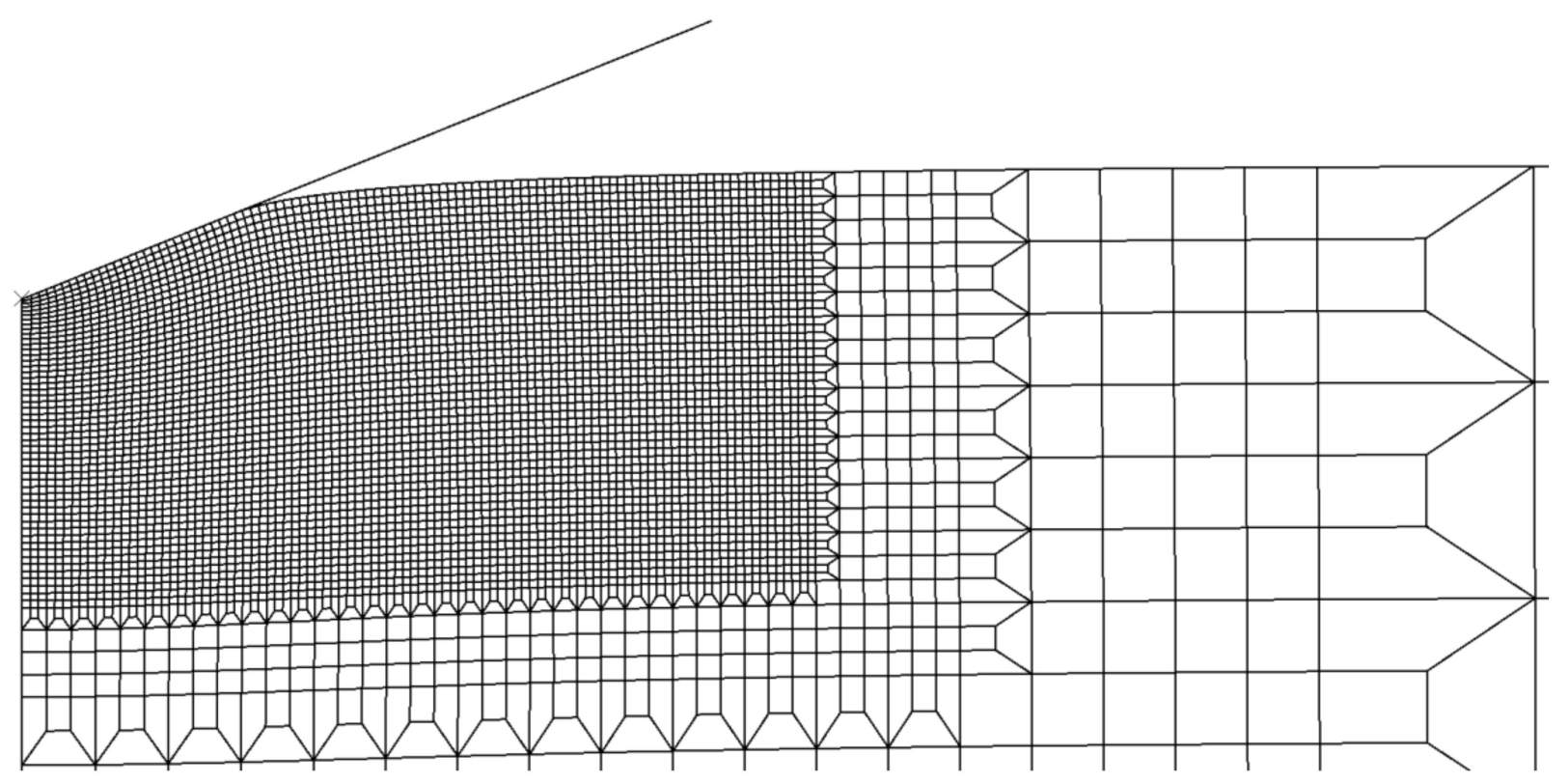

Figure 3. The finite element mesh used in the indentation simulations. Only the part of the mesh close to the region of contact is shown. The rigid conical indenter is also indicated.

Regarding the constitutive specification this has been stated above but is repeated here for clarity: Quasi-static conditions and classical Drucker-Prager elastoplasticity is relied upon. The investigation is focused on level III contact conditions if not clearly stated otherwise. Furthermore, frictionless contact is assumed.

Finite element analysis of axisymmetric elasto-plastic contact problems are nowadays considered to be a standard effort. Despite this, remembering that the present analysis also includes pressure-sensitivity in the material model, it was thought advisable to determine the reliability of the present numerical approach in particular concerning the contact problem. In doing so, present finite element results for cone indentation of an elastic material were compared with the corresponding analytical solution by Sneddon [1] reading

$$
P=2 E h^{2} \tan \theta /\left(\pi\left(1-v^{2}\right)\right)
$$

In (16), $h$ is as previously mentioned the normal indentation depth and $\theta$ is the semiincluded angle of the conical indenter and takes on the value $68^{\circ}$ (remembering that the angle between the indenter and the undeformed surface is presently fixed at $22^{\circ}$ ). The outcome of this comparison is depicted in Figure 4. Clearly the two solutions are basically on top of each other during the first half of the indentation simulation giving some confidence in the present finite element approach. The rather small differences between the two curves during the second half of indentation is due to the interference from the outer boundaries of the finite element mesh. This effect will be negligible at elastoplastic indentation as then the field variables falls off to zero much more rapidly than in the elastic case. A corresponding small deformation finite element analysis was also performed with 
the results being close (but not identical) to the large deformation ones. This indicates that large deformation effects on global indentation properties are relatively small.

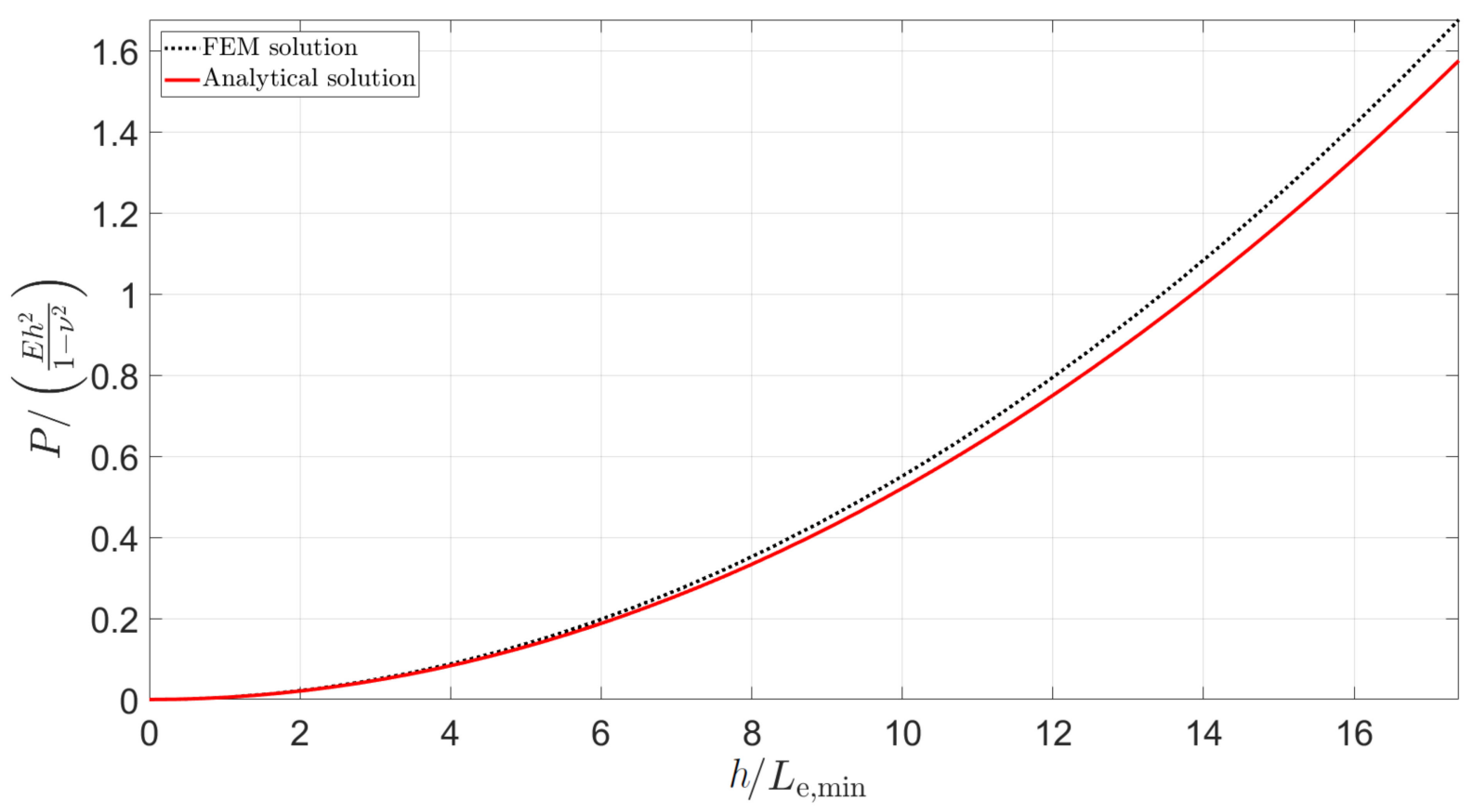

Figure 4. Analysis of conical indentation of a linearly elastic half-space. Comparison between present finite element results (accounting for large deformations) and the analytical solution by Sneddon [1] as detailed in Equation (16). In the figure, Le,min is the minimum element side length in the region of contact.

The number of elements in contact is an important feature when it comes to the accuracy of the solution. In [14] it was found that convergence (when defined as the numerical scatter being less than $2.5 \%$ ) of numerically determined indentation parameters required at least 15 elements within the contact region. Presently, the elastic numerical simulation discussed above was terminated (and relevant parameters evaluated) when the number of elements in contact was approximately 25 . In the plastic simulations this number was considerably higher due to piling up of material just outside the contact region (instead of sinking in occurring during the elastic problem). Accordingly, based on the findings in [14], problems concerning the accuracy of the solution due to this feature will be minor. Note that as mentioned above global indentation properties are in the present setting independent of indentation depth (and size of the contact area).

With the finite element procedure established it seems appropriate to also in this section determine the mechanical behavior of the cone indentation hardness $H_{c}$ in the spirit of Figure 1 but accounting for pressure-sensitivity. The results are shown in Figure 5 and clearly the fundamental behavior is the same regardless of the value on $\alpha$. It is of importance for the usefulness of the present analysis that rigid plastic conditions see Figure 1, are achieved at practically reasonable values, pertinent to essentially all metallic materials, on the Johnson parameter $\Lambda$ in Equation (1). Furthermore, it should be emphasized that the numerical results for classical von Mises plasticity in Figure 5 are very close to the well-established ones by Atkins and Tabor [6]. This gives further confidence in the present numerical approach.

Finally in this section, the values on the material properties, used in the simulations, should be presented and discussed. Some of these values are fixed during the entire analysis; this concerns $\sigma_{0}=1$ (normalizing value for stress quantities), $v=0.3$ and $\sigma r e p=\sigma\left(\varepsilon_{p}=0.18\right.$ ). The other material properties are determined from the chosen values on $\Lambda, \alpha$, and $n$. 


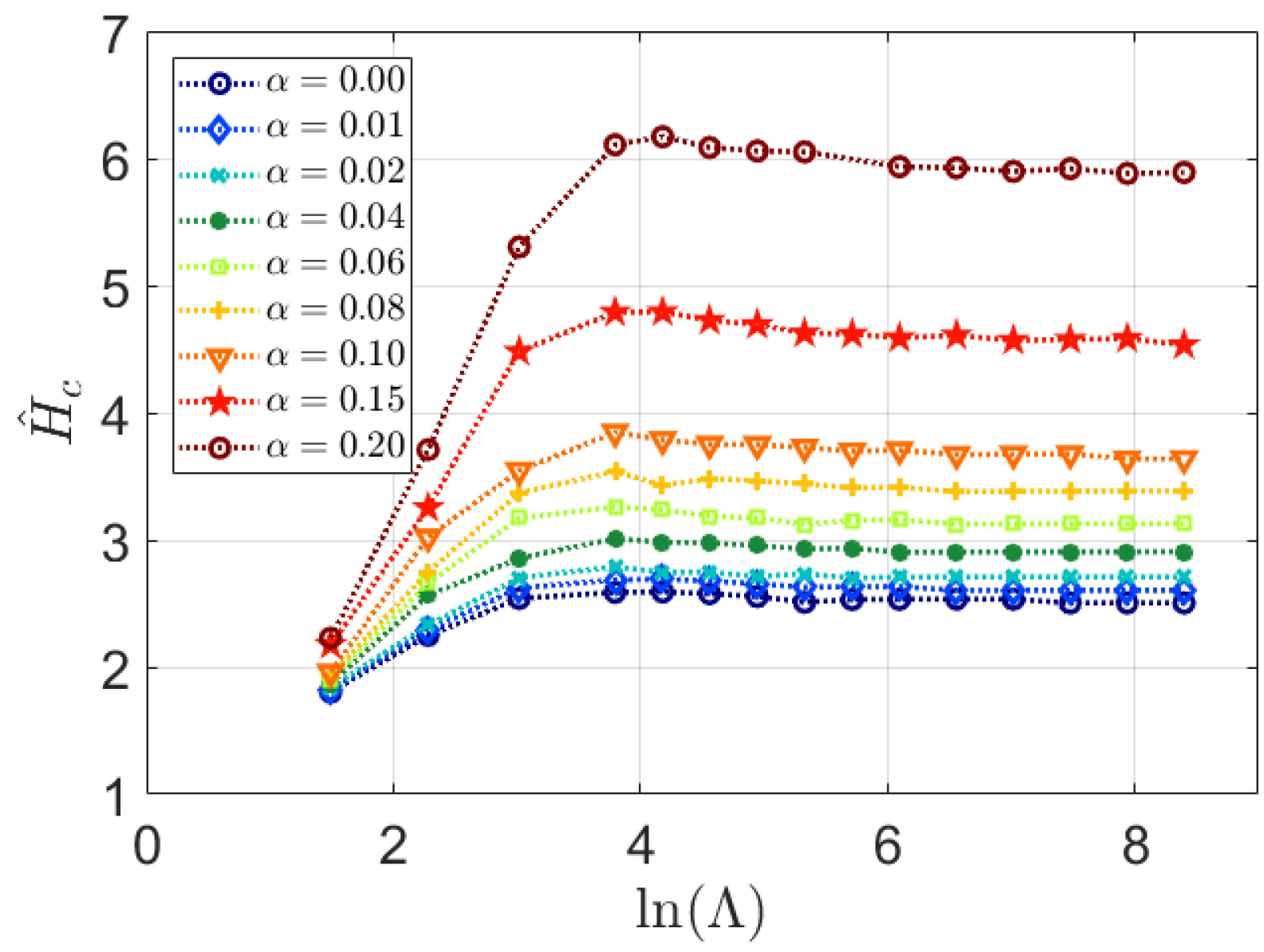

Figure 5. The normalized hardness $\hat{H} c\left(\hat{H} c=H c / \sigma\left(\varepsilon_{p}=0.18\right)\right)$ as function of the non-dimensional strain parameter $\Lambda$, suggested by Johnson [3,4], for different values on the pressure sensitivity factor $\alpha$. Ideally plastic behavior is assumed. Results from the finite element method (FEM) simulations of cone indentation.

\section{Results and Discussion}

The results below are pertinent to a theoretical and numerical analysis of sharp indentation testing of pressure-sensitive materials. In particular, cone indentation is considered and pressure-sensitivity is modelled using Drucker-Prager elastoplasticity. Rigid plastic (level III) contact is assumed to prevail, meaning that at least hardness results are not dependent on the elastic properties of the material. In the presentation below, the numerical (FEM) results will be used in order to verify and to improve the theoretical findings above, but also to describe the mechanical behavior of different indentation quantities not directly included in the theoretical analysis.

It seems appropriate to first investigate the influence from the plastic flow direction on the present results. In doing so, as already mentioned above, the two extremes, incompressible and associate plastic flow, are considered and compared using the finite element method. The outcome of this comparison is shown in Figure 6 for the normalized cone indentation hardness $\hat{H}_{c}$ for a particular value on the power law exponent $n(n=0.1)$ at rigid plastic conditions. Clearly the difference between the two sets of results is small; not only at small values on the pressure-sensitivity parameter $\alpha$ (which could be expected) but also at $\alpha=0.2$. Accordingly, this matter will not be dwelt upon further and associative plasticity is adhered to in all the calculations discussed below. 


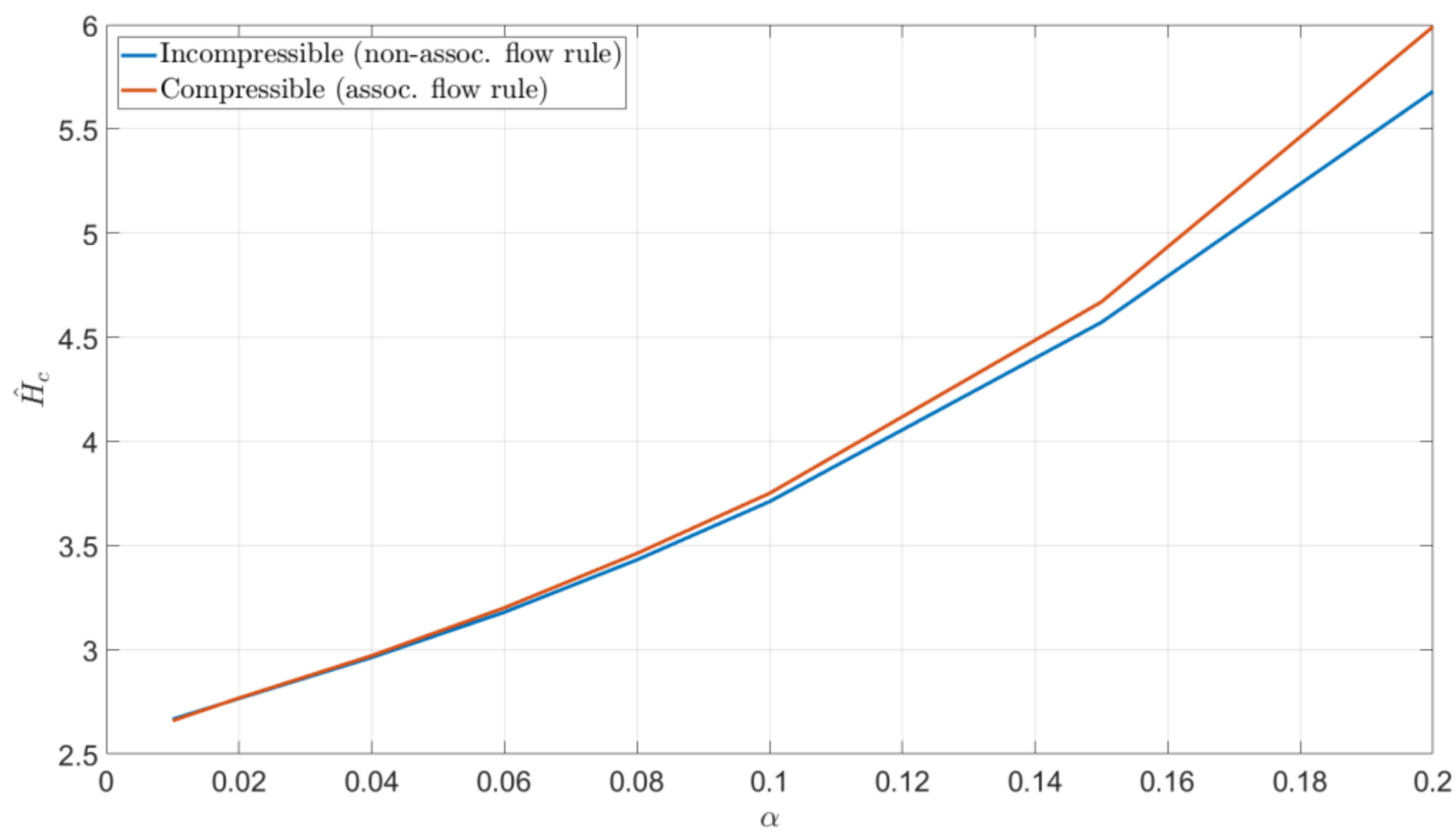

Figure 6. The normalized hardness $\hat{H} c\left(\hat{H} c=H c / \sigma\left(\varepsilon_{p}=0.18\right)\right)$ as function of the pressure sensitivity factor $\alpha$ at associative and incompressible plastic flow. Plastic strain hardening with a power law exponent $n=0.1$. Results from the FEM simulations of cone indentation.

A fundamental consequence of the assumptions made in the theoretical analysis concerns the separability of the influence from strain-hardening and pressure-sensitivity in the expressions for the material hardness. It therefore seems appropriate to investigate the accuracy of this feature. In doing so, FEM results pertinent to cone indentation are shown in Figures 7 and 8 for varying values on the power law exponent $n$ and the pressure-sensitivity parameter $\alpha$. In Figures 7 and 8 the normalized hardness defined in Equation (10) (specifically for cone indentation in Equation (13)) is written as

$$
\hat{H}_{c}=2.55 f(\alpha) g(n)
$$

assuming rigid plastic (level III) indentation and using the representative stress in Equation (3). The results in Figures 7 and 8 are indeed encouraging as it clearly shows that the influence from the two features is separable within good accuracy. It should be noted that Equation (13) suggests that

$$
g(n)=1
$$




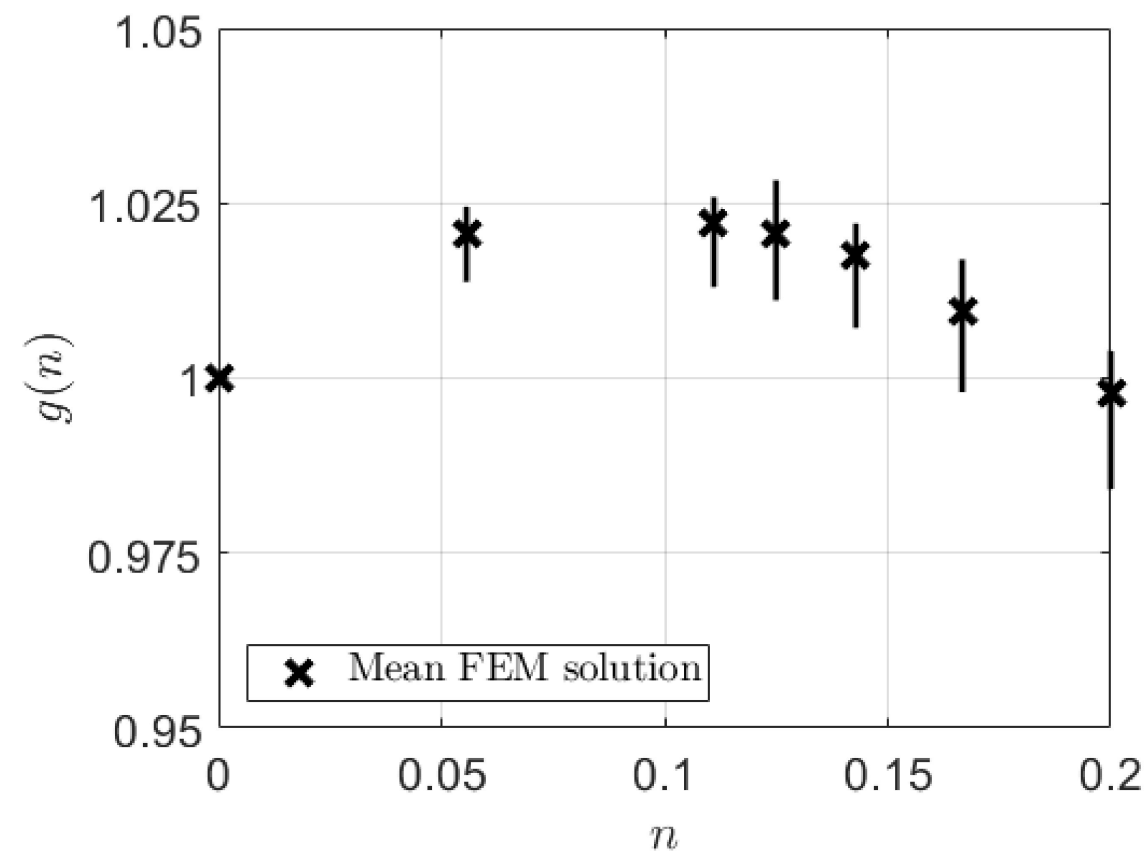

Figure 7. The function $g(n)$ in Equation (17) for values on the power law exponent $n$ up to 0.2. The results indicated are pertinent to the mean solution from axisymmetric FEM simulations (of cone indentation). The variation of results, at different values on the pressure sensitivity factor $\alpha$, is indicated.

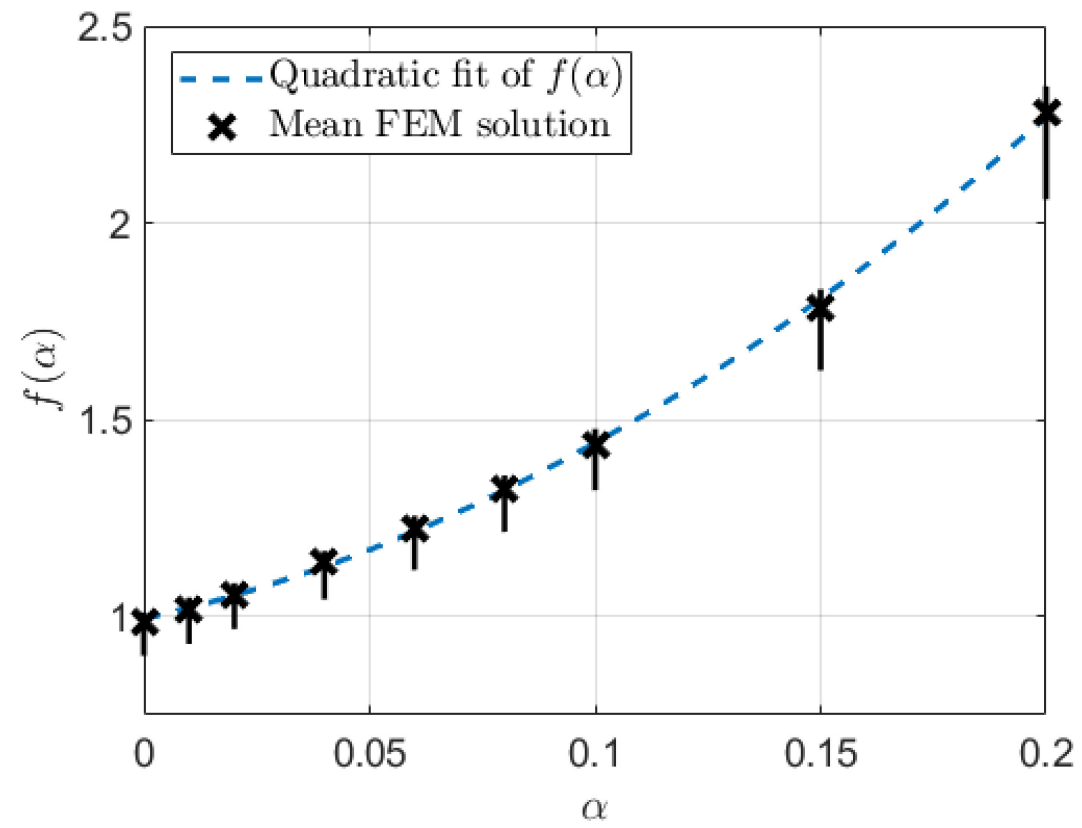

Figure 8. The function $f(\alpha)$ in Equation (17) for values on the pressure sensitivity factor $\alpha$ up to 0.2. The quadratic fit according to Equation (19) and the mean solution from axisymmetric FEM simulations (of cone indentation) are also shown as well as the variation of results for different values on the power law exponent $n$.

According to the results shown in Figure 7 this is accurately fulfilled at values of $n$ up to 0.2 . With the present choice of material parameters, strain-hardening becomes very high at increasing values of $n$ but if the material parameters were adjusted in order to achieve more practically reasonable strain-hardening $g(n)$ would be much closer to 1 also 
at $n>0.2$ or 0.25 , cf. $[23,27]$ (in particular Figure 9 in [27]), and this matter will not be investigated further.

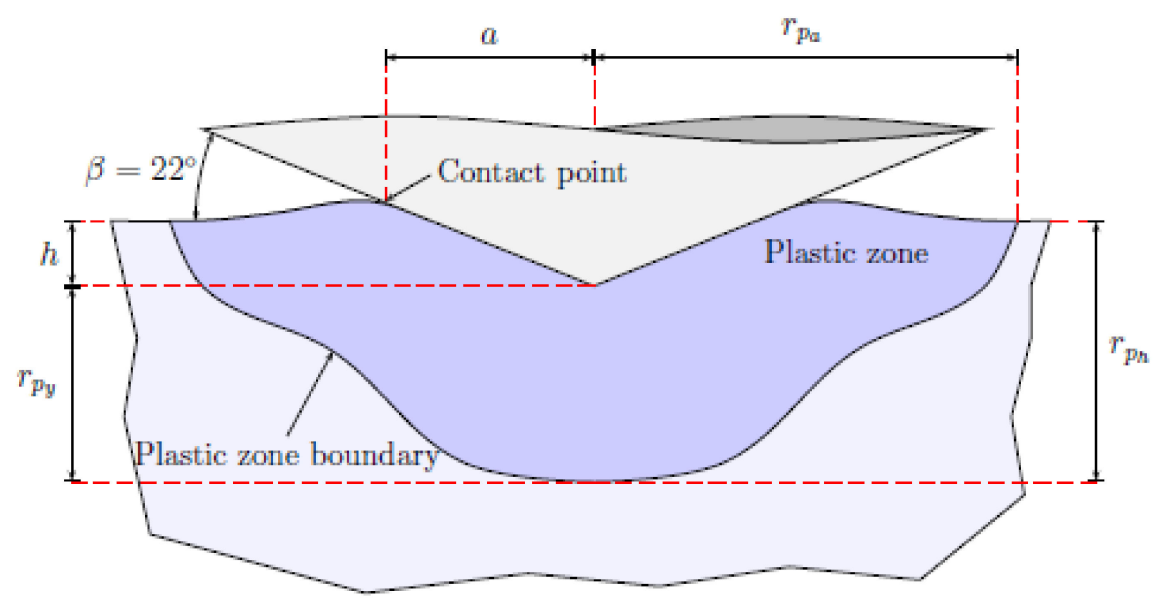

Figure 9. Plastic zone size parameters. In particular the quantity $r_{p y}\left(\bar{r}_{p y}\right.$ when normalized $)$ is defined.

With the separability of the problem now established it remains to investigate the functional form of $f(\alpha)$ in Equation (16). Based on the results shown in Figure 8 secondorder polynomial curve-fitting gives

$$
f(\alpha)=1.010+2.046 \alpha+21.87 \alpha^{2}
$$

for $\alpha$-values between 0 and 0.2 . This should in passing be compared to the presently theoretically derived expression in Equation (13) from which

$$
f(\alpha)=(1 / 3 \alpha)\left((1+2 \alpha)^{2} \bar{r}_{p y^{2 q}}-1\right) / 2.55
$$

However, in order to explicitly evaluate Equation (20) the size of the plastic zone at rigid plastic conditions, $\bar{r}_{p y}(\alpha)$, has to be determined. This is as already mentioned done by finite element calculations but also requires some further considerations to ensure an appropriate evaluation. As schematically shown in Figure 9 it is not immediately obvious how to represent the size of the plastic zone in a correct manner. Decisive results in this context have, however, been presented by Larsson and Olsson [28]. In this investigation it is shown that a constant value on the normalized plastic zone size parameter $\bar{r}_{p h}$ is reached immediately when entering level III contact conditions but not so for $\bar{r}_{p a}$ where elastic effects are noticeable also at $\ln \Lambda=8$. Accordingly, there is an agreement in mechanical (contact) behavior between the hardness $H$ and $\bar{r}_{p h}$ and the relative contact area and $\bar{r}_{p a}$. For this reason, $\bar{r}_{p h}$ is used to determine the plastic zone radius $\bar{r}_{p y}$ in Equation (20), and consequently in Equations (13) and (15). Indeed, regarding details, remembering the fact that large deformation theory is relied upon presently, $\bar{r}_{p y}=\bar{r}_{p h}-h, h$ being the indentation depth, is adhered to as shown in Figure 9. Second-order polynomial curve-fitting based on (rigid) ideally plastic finite element simulations then yields

$$
\bar{r}_{p y}=1.724+2.468 \alpha+13.61 \alpha^{2}
$$

It should be emphasized that the present results pertinent to von Mises plasticity are in very good agreement with the previous analyses; cf. [28].

Another matter that deserves, as already discussed above, additional consideration is the fact that in the separability stated in Equations (13) and (17) it is without further discussion assumed that $\bar{r}_{p y}=\bar{r}_{p y}(\alpha)$ neglecting the dependence of the strain-hardening exponent $n$. It has been shown in many investigations, cf. e.g., Mata et al. [29] that indeed the plastic zone size is dependent on the power law characteristics and accordingly an assumption 
$\bar{r}_{p y}=\bar{r}_{p y}(n, \alpha)$ would be theoretically more correct. However, in the corresponding analysis for von Mises plasticity it is well-known that the power law dependence is sufficiently described by determining $\bar{r}_{p y}$ at ideal plasticity and simply introducing a representative stress in Equations (3) and (4), cf. [3,4]. By adhering to this observation $\bar{r}_{p y}=\bar{r}_{p y}(\alpha)$ is the foundation for the analysis and this is validated by the agreement between theoretical and numerical results shown below.

Accordingly, two expressions for the material hardness, at indentation of pressuresensitive elastoplastic materials are presented. The first one is based on theoretical/analyt ical arguments and from Equations (13) and (21) the relation

$$
\hat{H}_{c}=(1 / 3 \alpha)\left((1+2 \alpha)^{2}\left(1.724+2.468 \alpha+13.61 \alpha^{2}\right)^{2 q}-1\right)
$$

can be determined. Second, based on Equations (17)-(19), the straightforward finite element simulations, drawing upon separability, also gives from curve-fitting a complete expression for the hardness yielding

$$
\hat{H}_{c}=2.55\left(1.010+2.046 \alpha+21.87 \alpha^{2}\right)
$$

In Figure 10, the predictions from Equations (22) and (23) are compared and clearly, the agreement is very satisfactory giving substantial confidence in both the present correlation approaches. It should be noted that according to Figure 10 the results based on Equation (22) yields $\hat{H}_{\mathcal{C}}$ slightly less than the established value of 2.55 at von Mises plasticity $(\alpha=0)$. Most likely this is due the fact that truly rigid plastic values on $\bar{r}_{p y}$ (at high values on $\Lambda$ ) does not exactly agree with the value of $\bar{r}_{p y}$ at the transition between level II and level III contact, where Equation (11) is evaluated for the present purpose, due to an overshoot of the explicit values of $\bar{r}_{p y}$ at the transition. This has been shown earlier in [28] (Figure 5 in [28]) and also for hardness values as shown in Figure 5. It should then be emphasized that the mechanical behavior is similar for $\bar{r}_{p y}$ and the material hardness. Accordingly, if this feature is accounted for even better agreement would be found between Equation (22) and the rigid plastic von Mises solution. This is not elaborated further though remembering that pressure-sensitivity is an issue here.

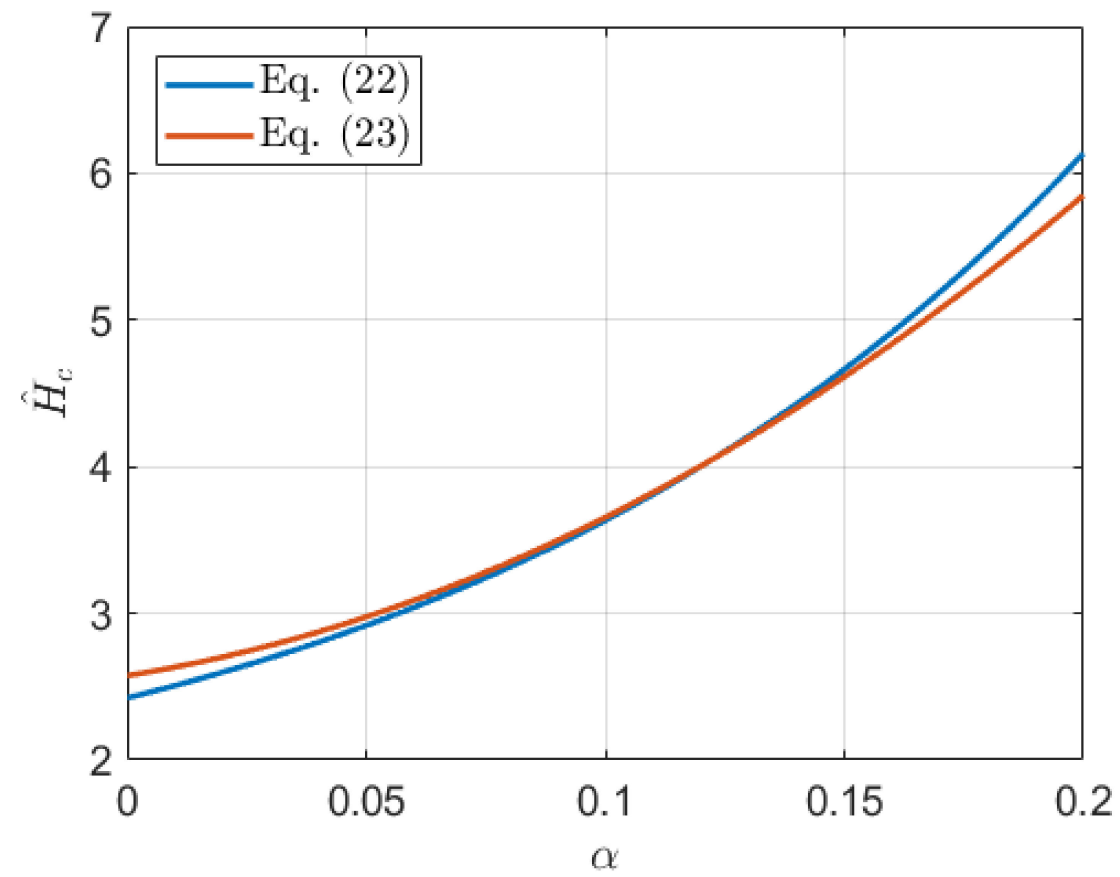

Figure 10. Comparison between the results given by Equations (22) and (23) for different values on the pressure sensitivity factor $\alpha$. 
It should be emphasized that the present approach can be used to confirm the empirical results for cone indentation [6] and correspondingly for pyramid indentation [2] via Equations (14) and (15). It is somewhat surprising that the present semi-theoretical approach has not been used previously to confirm the classical empirical results in [2] and [6]. In particular so as sufficient numerical capacity has been available for a long time in order to accurately determine the plastic zone radius $\bar{r}_{\text {py }}$.

Having established the validity of the present approach for two-dimensional (axisymmetric) indentation, it should be remembered that the corresponding expression for Berkovich, and Vickers indentation is given by Equation (15). According to the previous discussion, the size of the plastic zone is taken to be the same as for cone indentation. However, as mentioned above, the validity of Equation (15) will be investigated in more detail in a parallel study [25] also including indenter geometry and frictional effects and this matter will not be dwelled upon further in the present context.

For completeness, results pertinent to the relative contact area $c^{2}$, as defined in Equation (5), will also be presented. The first part of these results is depicted in Figure 11 and as can be seen, the qualitative behavior of this parameter is the same regardless if pressure-sensitivity is accounted for or not. In particular, as is the case at classical von Mises plasticity, elastic effects are present also at high values on $\Lambda$ and rigid plastic conditions are achieved only at essentially unrealistic values on this quantity. Nevertheless, rigid plastic results for $c^{2}$ can serve as a guideline at material characterization at classical von Mises plasticity and it certainly deserves to investigate this matter further also in the present situation. This was investigated in the context of separability at rigid plasticity, in the spirit of Equation (17) for hardness values, and the result is shown in Figure 12. Clearly, separability is not an accurate assumption for pressure-sensitive materials and any attempt to use $c^{2}$-values for correlation with material properties is very complicated and not elucidated (previously or here) in the literature. Accordingly, this will not be attempted presently.

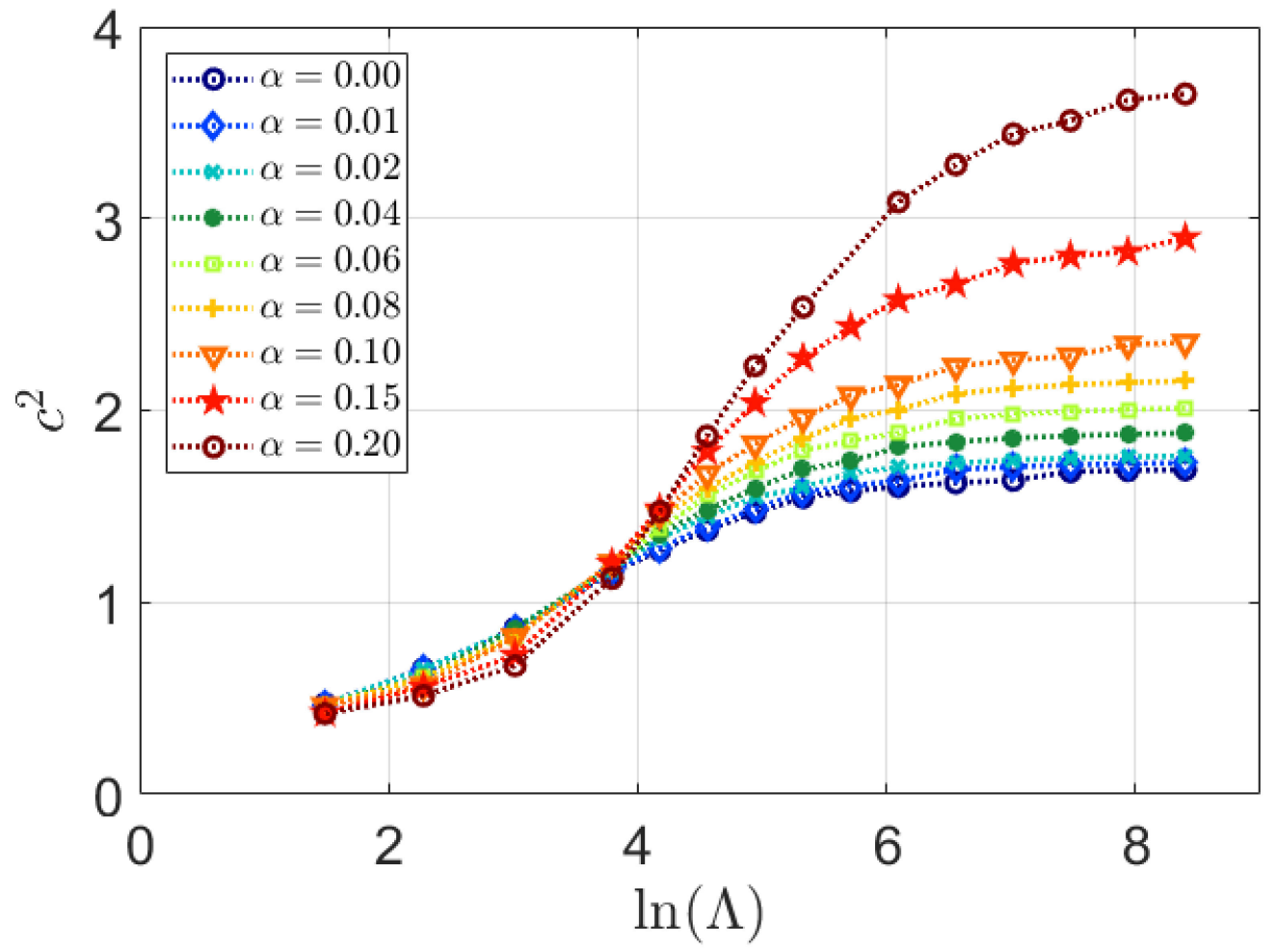

Figure 11. The relative contact area $c^{2}$ as function of the non-dimensional strain parameter $\Lambda$, suggested by Johnson $[3,4]$, for different values on the pressure sensitivity factor $\alpha$. Ideally plastic behavior is assumed. Results from the FEM simulations of cone indentation. 


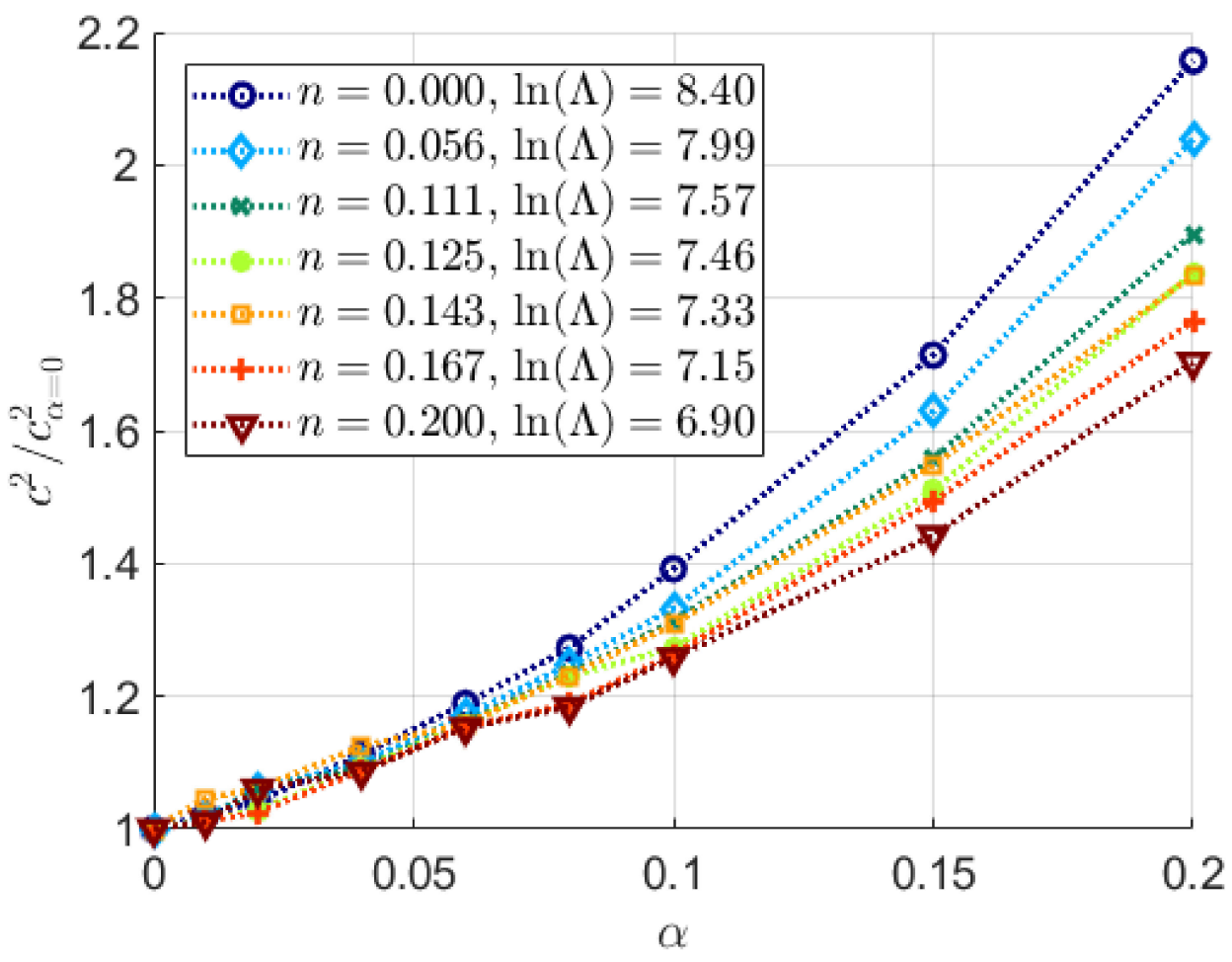

Figure 12. Normalized relative contact area, $c^{2}(\alpha, n) / c^{2}(\alpha) n=0$, for values on the pressure sensitivity factor $\alpha$ up to 0.2 and different values on the power law exponent $n$. The $c^{2}$-values are determined from the FEM-simulations (of cone indentation) at rigid plastic conditions with $\Lambda$-values indicated in the figure.

As a final comment it should be emphasized that even though the relations above will provide valuable information about the mechanical properties of pressure-sensitive materials, they are not sufficient for determining the material properties in a complete manner. This is so even if only the plastic properties of the material (corresponding to rigid plastic indentation behavior) are of interest. For this reason, it is of course unfortunate that additional information, solely related to the plastic behavior of the material, cannot be given by the parameter $c^{2}$. Accordingly, as it stands now, the present results should be seen as a complement to other types of mechanical testing for material characterization, and not as a complete alternative. Having said this, it is clear that the present results are of fundamental practical value as for the first time, to the authors knowledge, a relation for pressuresensitive materials that match the Tabor formula in Equation (2), for classical elastoplastic materials, has been presented. In this context it should be emphasized that Equation (22) is not a result of empirical considerations but instead, rests on a solid theoretical foundation.

\section{Conclusions}

Correlation of global indentation quantities have been investigated presently pertinent to level III (rigid plastic) indentation of materials constitutively described by DruckerPrager elastoplasticity. The most important results can be summarized as follows:

Two closed form relations for the indentation hardness are presented based on (1) a fundamental mechanical analysis accounting for deformation characteristics in the contact region and (2) curve-fitting of results from present numerical findings. The two different relations show very good agreement.

The closed form relations are specifically presented for cone indentation but an extension to pyramid indentation is detailed.

FEM results for the relative contact area at indentation show that this parameter is, as in case of von Mises plasticity, very much influenced by the elastic effects and correlation using this quantity is therefore not attempted here. 
The derived relations are in direct correspondence to the famous Tabor relation, pertinent to von Mises plasticity, but for Drucker-Prager plasticity. To the authors knowledge such formulas have not been presented previously. In short, this constitutes a new tool for the proper characterization of pressure-sensitive materials.

Author Contributions: All authors were involved in every aspect of the article. All authors have read and agreed to the published version of the manuscript.

Funding: This research received no external funding.

Institutional Review Board Statement: Not applicable for studies not involving humans.

Informed Consent Statement: Not applicable for studies not involving humans.

Conflicts of Interest: The authors declare no conflict of interest.

\section{References}

1. Sneddon, I.N. The relation between load and penetration in the axisymmetric Boussinesq problem for a punch of arbitrary profile. Int. J. Eng. Sci. 1965, 3, 47-57. [CrossRef]

2. Tabor, D. Hardness of Metals; Cambridge University Press: Cambridge, UK, 1951.

3. Johnson, K.L. The correlation of indentation experiments. J. Mech. Phys. Solids 1970, 18, 115-126. [CrossRef]

4. Johnson, K.L. Contact Mechanics; Cambridge University Press: Cambridge, UK, 1985.

5. Drucker, D.C.; Prager, W. Soil mechanics and plastic analysis for limit design. Q. Appl. Math. 1952, 10, 157-165. [CrossRef]

6. Atkins, A.G.; Tabor, D. Plastic indentation in metals with cones. J. Mech. Phys. Solids 1965, 13, 149-164. [CrossRef]

7. Bhattachharya, A.K.; Nix, W.D. Finite element simulation of indentation experiments. Int. J. Solids Struct. 1988, 24, 881-891. [CrossRef]

8. Bhattachharya, A.K.; Nix, W.D. Analysis of elastic and plastic deformation associated with indentation testing of thin films on substrates. Int. J. Solids Struct. 1988, 24, 1287-1298. [CrossRef]

9. Laursen, T.A.; Simo, J.C. A study of the mechanics of microindentation using finite elements. J. Mater. Res. 1992, 7, 618-626. [CrossRef]

10. Giannakopoulos, A.E.; Larsson, P.-L.; Vestergaard, R. Analysis of Vickers indentation. Int. J. Solids Struct. 1994, 31, 2679-2708. [CrossRef]

11. Larsson, P.-L.; Giannakopoulos, A.E.; Söderlund, E.; Rowcliffe, D.J.; Vestergaard, R. Analysis of Berkovich indentation. Int. J. Solids Struct. 1996, 33, 221-248. [CrossRef]

12. Mesarovic, S.D.; Fleck, N.A. Spherical indentation of elastic-plastic solids. Int. J. Solids Struct. 2000, 37, 7071-7091. [CrossRef]

13. Mesarovic, S.D.; Fleck, N.A. Frictionless indentation of dissimilar elastic-plastic spheres. Proc. R. Soc. Lond. A 1999, 455, 2707-2728. [CrossRef]

14. Larsson, P.-L. Investigation of sharp contact at rigid plastic conditions. Int. J. Mech. Sci. 2001, 43, 895-920. [CrossRef]

15. Giannakopoulos, A.E.; Larsson, P.-L. Analysis of pyramid indentation of pressure-sensitive hard metals and ceramics. Mech. Mater. 1997, 25, 1-35. [CrossRef]

16. Narasimhan, R. Analysis of indentation of pressure-sensitive plastic solids using the expanding cavity model. Mech. Mater. 2004, 36, 633-645. [CrossRef]

17. Zhang, H.W.; Subhash, G.; Jing, X.N.; Kecskes, L.J.; Dowding, R.J. Evaluation of hardness-yield strength relationships for bulk metallic glasses. Philos. Mag. Lett. 2006, 86, 333-345. [CrossRef]

18. Fornell, J.; Concustell, A.; Surinach, S.; Li, W.H.; Cuadrado, N.; Gebert, A.; Baro, M.D.; Sort, J. Yielding and intrinsic plasticity of Ti-Zr-Ni-Cu-Be bulk metallic glasses. Int. J. Plast. 2009, 25, 1540-1559. [CrossRef]

19. Giannakopoulos, A.E.; Zisis, T. Analysis of Knoop indentation of cohesive frictional materials. Mech. Mater. 2013, 57, 53-74. [CrossRef]

20. Needleman, A.; Tvergaard, V.; van der Giessen, E. Indentation of elastically soft and plastically compressible solids. Acta Mech. Sin. 2015, 31, 473-480. [CrossRef]

21. Larsson, P.-L. Modelling of sharp indentation experiments: Some fundamental issues. Philos. Mag. 2006, 86, 5155-5177. [CrossRef]

22. Bishop, R.F.; Hill, R.; Mott, N.F. The theory of indentation and hardness test. Proc. Phys. Soc. 1945, 57, 147-159. [CrossRef]

23. Larsson, P.-L. On the mechanical behavior of global parameters in material characterization by sharp indentation testing. J. Test. Eval. 2004, 32, 310-321. [CrossRef]

24. Khrushchov, M.M.; Berkovich, E.S. Methods of Determining the Hardness of Very Hard Materials: The Hardness of Diamond. Ind. Diam. Rev. 1951, 11, 42-49.

25. Faleskog, J.; Dahlberg, C.F.O.; Larsson, P.-L. Influence of friction and geometry on the correlation of sharp indentation testing. Work in progress.

26. Dassault Systemes Simulia Corporation. Abaqus. In Analysis User's Manual Version 6.14-2; Dassault Systemes Simulia Corporation: Providence, RI, USA, 2014. 
27. Olsson, E.; Larsson, P.-L. A unified correlation model describing global properties at elastoplastic sharp indentation contact. Tribol. Int. 2016, 103, 252-260. [CrossRef]

28. Larsson, P.-L.; Olsson, E. Plastic zone size at sharp indentation contact of classical elastic-plastic materials: Behavior at linear strain hardening. J. Test. Eval. 2017, 45, 1854-1861. [CrossRef]

29. Mata, M.; Casals, O.; Alcala, J. The plastic zone size in indentation experiments: The analogy with the expansion of a spherical cavity. Int. J. Solids Struct. 2006, 43, 5994-6013. [CrossRef] 